ANNALES

POLONICI MATHEMATICI

$80(2003)$

\title{
Analytic continuation for some classes of separately analytic functions of real variables
}

\author{
by NGuYen Thanh Van (Toulouse)
}

En hommage à Monsieur le Professeur Józef Siciak

Abstract. For functions that are separately solutions of an elliptic homogeneous PDE with constant coefficients, we prove an analogue of Siciak's theorem for separately holomorphic functions.

\section{Definitions and the principal result}

Definition. Let $Q$ be an elliptic homogeneous polynomial of $N$ real variables. A complex function $f$ defined in an open subset $\Omega$ of $\mathbb{R}^{N}$ is called $Q$-analytic if it is real-analytic and satisfies

$$
Q\left(\frac{\partial}{\partial x_{1}}, \ldots, \frac{\partial}{\partial x_{N}}\right) f=0 .
$$

The set of $Q$-analytic functions in $\Omega$ will be denoted $A Q(\Omega)$.

Definition. Let $D_{j}$ be an open subset of $\mathbb{R}^{N_{j}}$ and $E_{j}$ a compact subset of $D_{j}, j=1, \ldots, p$. Put

$\Gamma=\Gamma\left(D_{1}, \ldots, D_{p} ; E_{1}, \ldots, E_{p}\right)=\left(D_{1} \times E_{2} \times E_{p}\right) \cup \ldots \cup\left(E_{1} \times \ldots \times E_{p-1} \times D_{p}\right)$.

Let $Q_{j}(1 \leq j \leq p)$ be an elliptic homogeneous polynomial of $N_{j}$ real variables. A function $f: \Gamma \rightarrow \mathbb{C}$ is called $\left(Q_{1}, \ldots, Q_{p}\right)$-separately analytic if for every fixed $j \in\{1, \ldots, p\}$ and every fixed $x \in \prod_{k=1}^{p} E_{k}$, the function $t \mapsto f\left(\breve{x}_{j}, t\right)$ is $Q_{j}$-analytic in $D_{j}$, where $\breve{x}_{j}=\left(x_{1}, \ldots, x_{j-1}, x_{j+1}, \ldots, x_{p}\right)$.

Problem. Under some hypothesis on $E_{j}$ and $D_{j}$, find an open neighbourhood $V$ of $\Gamma$ such that every $\left(Q_{1}, \ldots, Q_{p}\right)$-separately analytic function on $\Gamma$ can be analytically continued to a real-analytic function on $V$.

We shall need some extremal functions studied by Hécart [2]-[4], first introduced by Zahariuta [11].

2000 Mathematics Subject Classification: 31B05, 32A10, 30E10.

Key words and phrases: separately analytic, $Q$-analytic, analytic continuation. 
Definition. Let $D$ be an open subset of $\mathbb{R}^{N}, E$ a compact set in $D$ and $Q$ an elliptic homogeneous polynomial of $N$ real variables. We define

$$
\begin{aligned}
K_{Q, \varepsilon}(D, E, x) & =\sup \{\alpha \log |u(x)|: u \in A Q(D), 0<\alpha<\varepsilon, \\
\left.\|u\|_{E} \leq 1,\|u\|_{D} \leq \exp (1 / \alpha)\right\} \quad(\varepsilon>0), & \\
\chi_{Q, \varepsilon}(D, E, x) & =\limsup _{x^{\prime} \rightarrow x} K\left(D, E, x^{\prime}\right), \\
\chi_{Q, 0}(D, E, x) & =\lim _{\varepsilon \rightarrow 0} \chi_{Q, \varepsilon}(D, E, x), \\
\chi_{Q}(D, E, x) & =\lim _{s \rightarrow \infty} \chi_{Q, 0}\left(D_{s}, E, x\right),
\end{aligned}
$$

where $E \subset D_{s} \Subset D_{s+1}$ and $\bigcup D_{s}=D$.

Theorem 1. If $D_{j}$ is connected and $E_{j}$ is L-regular in $\mathbb{C}^{N_{j}}(1 \leq j \leq p)$, then every $\left(Q_{1}, \ldots, Q_{p}\right)$-separately analytic function on $\Gamma$ can be analytically continued to

$$
\widehat{\Gamma}=\left\{\left(x_{1}, \ldots, x_{p}\right) \in D_{1} \times \ldots \times D_{p}: \sum_{j=1}^{p} \chi_{Q_{j}}\left(D_{j}, E_{j}, x_{j}\right)<1\right\} .
$$

REMARKs. $\bullet$ We consider $\mathbb{R}^{N}$ as the real part of $\mathbb{C}^{N}$.

- A compact set $E$ in $\mathbb{C}^{N}$ is called $L$-regular if its Siciak extremal function $\phi_{E}$ is continuous; for details we refer to Klimek's book [5].

- The $L$-regularity of $E_{j}$ implies that $\widehat{\Gamma}$ is an open neighbourhood of $\Gamma$.

\section{Preliminary results}

Proposition 1. Let $Q$ be an elliptic homogeneous polynomial in $\mathbb{R}^{N}$. For every $r>0$, there exist $\varrho=\varrho(r, Q)>0$ and $C=C(r, Q)$ such that every $Q$-analytic function $f$ in the real ball $B(0, r)$ is analytically continuable to the complex ball $\widehat{B}(0, \varrho)$, and the continuation $\widehat{f}$ satisfies $\|\widehat{f}\|_{\widehat{B}} \leq C\|f\|_{B}$.

Proposition 2. Let $\Omega_{j}$ be a bounded pseudoconvex open set in $\mathbb{C}^{N_{j}}$ and $E_{j}$ a Borel set in $\Omega_{j}(1 \leq j \leq p)$. If $f$ is separately holomorphic on the crossed set

$$
X=\left(\Omega_{1} \times E_{2} \times \ldots \times E_{p}\right) \cup \ldots \cup\left(E_{1} \times \ldots \times E_{p-1} \times \Omega_{p}\right),
$$

then $f$ is holomorphically continuable to

$$
\widehat{X}=\left\{\left(z_{1}, \ldots, z_{p}\right) \in \Omega_{1} \times \ldots \times \Omega_{p}: \sum_{j=1}^{p} \omega^{*}\left(\Omega_{j}, E_{j}, z_{j}\right)<1\right\}
$$

where $\omega^{*}\left(\Omega_{j}, E_{j}, \cdot\right)$ is the $(0,1)$-psh extremal function associated to $E_{j}$ and $\Omega_{j}$.

Proposition 3. Let $D$ be a bounded domain in $\mathbb{R}^{N}, E$ an L-regular compact subset of $D$ and $\mu=\left(d d^{c} \log \phi_{E}\right)^{N}$ the Monge-Ampère measure 
on E. Let $Q$ be an elliptic homogeneous polynomial in $\mathbb{R}^{N}$, and put

$$
L_{Q}^{2}(D)=A Q(D) \cap L^{2}(D, \lambda),
$$

where $\lambda$ is the Lebesgue $N$-dimensional measure. Then there exists an orthogonal basis $\left(B_{k}\right)$ of the Hilbert space $L_{Q}^{2}(D)$ such that

(i) $\sum \mu_{k}^{-\delta}<\infty$ for all $\delta>0$, with $\mu_{k}=\left(\int_{D}\left|B_{k}\right|^{2} d \lambda\right)^{1 / 2}$ increasing to $\infty$,

(ii) $\int_{E} B_{k} \bar{B}_{l} d \mu= \begin{cases}0 & \text { if } k \neq l, \\ 1 & \text { if } k=l,\end{cases}$

(iii) for every $\varepsilon>0$ there exists $C=C(\varepsilon)$ such that $\left\|B_{k}\right\|_{E}:=\sup _{E}\left|B_{k}\right|$ $\leq C \mu_{k}^{\varepsilon}$.

Proposition 1 is taken from Armitage, Bagby and Gauthier [1].

Proposition 2, a general version of the Siciak-Zahariuta theorem, is from $[10]$.

Proof of Proposition 3. It is easy to verify that

$$
L_{Q}^{2}(D) \hookrightarrow A Q(D) \hookrightarrow L^{2}(E, \mu),
$$

where $\hookrightarrow$ means a compact imbedding defined by the restriction operator. Because $A Q(D)$ is a nuclear Fréchet space with the compact convergence topology [3], a theorem of Mityagin [7] gives the existence of an orthogonal basis $\left(B_{k}\right)$ of $L_{Q}^{2}(D)$ satisfying (i) and (ii).

We now deduce (iii). Put

$$
\widehat{D}=\bigcup_{z \in D} \widehat{B}\left(z, \varrho_{z}\right) \quad \text { with } \quad \varrho_{z}=\varrho(\operatorname{dist}(z, \partial D), Q) .
$$

Following Proposition 1, we can continue $B_{k}$ to a holomorphic function $\widehat{B}_{k}$ in $\widehat{D}$ such that for every $K$ compact $\subset \widehat{D}$,

$$
\left\|\widehat{B}_{k}\right\|_{K} \leq M(K) \mu_{k}, \quad \forall k .
$$

From this inequality we see that the family $\left(\log \left|B_{k}\right| / \log \mu_{k}\right)$ is locally upper bounded in $\widehat{B}$. We put

$$
W=\limsup \frac{\log \left|B_{k}\right|}{\log \mu_{k}}, \quad W^{*}=\operatorname{reg} \sup W .
$$

A known argument [10] yields

$$
W^{*}(z) \leq 0, \quad \forall z \in E \backslash E^{\prime},
$$

for some $E^{\prime}$ with $\mu\left(E^{\prime}\right)=0$. By Levenberg [6], $E \backslash E^{\prime}$ satisfies the $\left(L_{0}\right)$ condition of Leja at every point of $E$. Because of the polynomial convexity of $E$ in $\mathbb{C}^{N}$ and the fact that $W^{*} \in \operatorname{PSH}(\widehat{D})$ with $\widehat{D} \supset E$, we have $W^{*} \leq 0$ on $E$, by [8, Th. 2]. This last inequality and the classical Hartogs Lemma imply (iii). 


\section{Proof of Theorem 1}

3.1. For every $j=1, \ldots, p$, we choose a domain $D_{j}^{\prime}$ such that $E_{j} \subset D_{j}^{\prime}$ $\Subset D_{j}$. Because $\bar{D}_{j}$ is a polynomially convex subset of $\widehat{D}_{j}$, it has a bounded pseudoconvex open neighbourhood $\Omega_{j}$ in $\widehat{D}_{j}$ (for the definition of $\widehat{D}_{j}$, see the beginning of the proof of Proposition 3 ).

For fixed $\left(x_{1}, \ldots, x_{p}\right) \in E_{1} \times \ldots \times E_{p}$, let $\widehat{f}\left(\breve{x}_{j}, \cdot\right)$ be the analytic continuation of $f\left(\breve{x}_{j}, \cdot\right)$ to $\widehat{D}_{j}$; gluing up these functions, we obtain a separately holomorphic function $\widehat{f}$ on the crossed set

$$
X=\left(\Omega_{1} \times E_{2} \times \ldots \times E_{p}\right) \cup \ldots \cup\left(E_{1} \times \ldots \times E_{p-1} \times \Omega_{p}\right) .
$$

By Proposition 2, there exists a holomorphic function $g$ on

$$
\widehat{X}=\left\{\left(z_{1}, \ldots, z_{p}\right) \in \Omega_{1} \times \ldots \times \Omega_{p}: \sum_{j=1}^{p} \omega^{*}\left(\Omega_{j}, E_{j}, x_{j}\right)<1\right\},
$$

with $g=\widehat{f}$ on $X$.

In the next paragraphs, it is important to note that $\widehat{X}$ is an open neighbourhood in $\mathbb{C}^{N_{1}+\ldots+N_{p}}$ of $\left(\overline{D_{1}^{\prime}} \times E_{2} \times \ldots \times E_{p}\right) \cup \ldots \cup\left(E_{1} \times \ldots \times E_{p-1} \times \overline{D_{p}^{\prime}}\right)$.

3.2. For every $j=1, \ldots, p$, let $\left(B_{k}^{j}\right)_{k=0,1, \ldots}$ be the doubly orthogonal basis of $L_{Q_{j}}^{2}\left(D_{j}^{\prime}\right)$ given by Proposition 3 , and $\mu^{j}$ the measure $\left(d d^{c} \log \phi_{E_{j}}\right)^{N_{j}}$. For $\alpha=\left(\alpha_{1}, \ldots, \alpha_{p}\right) \in \mathbb{N}^{p}$, put

$$
C_{\alpha}=\int_{E_{1} \times \ldots \times E_{p}} f\left(x_{1}, \ldots, x_{p}\right) \overline{B_{\alpha_{1}}^{1}\left(x_{1}\right)} \ldots \overline{B_{\alpha_{p}}^{p}\left(x_{p}\right)} d \mu^{1}\left(x_{1}\right) \wedge \ldots \wedge d \mu^{p}\left(x_{p}\right) .
$$

We have $\left|C_{\alpha}\right| \leq M / \mu_{\alpha_{j}}^{j}$, with $M$ independent of $j$ and $\alpha_{j}$, and

$$
\mu_{\alpha_{j}}^{j}=\left(\int_{D_{j}^{\prime}}\left|B_{\alpha_{j}}^{j}\right|^{2} d \lambda^{j}\right)^{1 / 2} \quad\left(\lambda^{j}=\text { Lebesgue measure in } \mathbb{R}^{N_{j}}\right) .
$$

In fact, this inequality results, via obvious estimates, from the following identities:

$$
C_{\alpha}=\int_{\breve{E}_{j}}\left(\int_{E_{j}} f\left(x_{1}, \ldots, x_{p}\right) d \mu^{j}\left(x_{j}\right)\right) \prod_{k \neq j} B_{\alpha_{k}}^{k}\left(x_{k}\right) \bigwedge_{k \neq j} d \mu^{k}\left(x_{k}\right),
$$

where $\breve{E}_{j}=E_{1} \times \ldots \times E_{j-1} \times E_{j+1} \times \ldots \times E_{p}$, and

$$
\int_{E_{j}} f\left(x_{1}, \ldots, x_{p}\right) \overline{B_{\alpha_{j}}^{j}\left(x_{j}\right)} d \mu^{j}\left(x_{j}\right)=\left(\mu_{\alpha_{j}}^{j}\right)^{-2} \int_{D_{j}^{\prime}} f\left(x_{1}, \ldots, x_{p}\right) \overline{B_{\alpha_{j}}^{j}\left(x_{j}\right)} d \lambda^{j}\left(x_{j}\right) .
$$


3.3. Now we prove the local absolute uniform convergence of the series $\sum_{\alpha \in \mathbb{N} p} C_{\alpha} B_{\alpha}(z)$ with $B_{\alpha}(z)=B_{\alpha_{1}}^{1}\left(z_{1}\right) \ldots B_{\alpha_{p}}^{p}\left(z_{p}\right)$ in the open set

$$
\widehat{\Gamma}^{\prime}=\left\{\left(x_{1}, \ldots, x_{p}\right) \in D_{1}^{\prime} \times \ldots \times D_{p}^{\prime}: \sum_{j=1}^{p} \chi_{Q_{j}}\left(D_{j}^{\prime}, E_{j}, x_{j}\right)<1\right\} .
$$

Let $a=\left(a_{1}, \ldots, a_{p}\right) \in \widehat{\Gamma}^{\prime}$. We can choose domains $D_{j}^{\prime \prime}$ and $\theta_{j}>0(j=$ $1, \ldots, p)$ such that

- $\sum \theta_{j}<1$,

- $E_{j} \subset D_{j}^{\prime \prime} \Subset D_{j}^{\prime}$,

• $a_{j} \in D_{j}^{\prime \prime}\left(\theta_{j}\right):=\left\{x \in D_{j}^{\prime \prime}: \chi_{Q_{j}}\left(D_{j}^{\prime \prime}, E_{j}, x\right)<\theta_{j}\right\}$.

We now choose $\theta_{j}^{\prime}>\theta_{j}$ with $\sum \theta_{j}^{\prime}=1$. From the estimate of $\left|C_{\alpha}\right|$ given in 3.2 , we deduce that

$$
\left|C_{\alpha}\right| \leq M\left(\mu_{\alpha_{1}}^{1}\right)^{-\theta_{1}^{\prime}} \ldots\left(\mu_{\alpha_{p}}^{p}\right)^{-\theta_{p}^{\prime}}
$$

Let $V_{j}$ be a compact neighbourhood of $a_{j}$ in $D_{j}^{\prime \prime}\left(\theta_{j}\right)$. By the two-constants theorem [2], [3], for every $\varepsilon \in] 0,1-\theta_{j}\left[\right.$ there exists $C=C\left(V_{j}, \varepsilon\right)$ such that

$$
\|u\|_{V_{j}} \leq C\|u\|_{E_{j}}^{1-\theta_{j}-\varepsilon}\|u\|_{D_{j}^{\prime \prime}}^{\theta_{j}+\varepsilon}, \quad \forall u \in A Q\left(D_{j}^{\prime \prime}\right)
$$

From Proposition 3(iii) and the obvious fact

$$
\left\|B_{\alpha_{j}}^{j}\right\|_{D_{j}^{\prime \prime}} \leq C^{\prime} \mu_{\alpha_{j}}^{j}
$$

we have, for every $\varepsilon \in] 0, \inf \theta_{j}[$,

$$
\left\|B_{\alpha}\right\|_{V} \leq C^{\prime \prime} \prod_{j=1}^{p}\left(\mu_{\alpha_{j}}^{j}\right)^{\varepsilon\left(1-\theta_{j}-\varepsilon\right)}\left(\mu_{\alpha_{j}}^{j}\right)^{\theta_{j}+\varepsilon}
$$

where $V=V_{1} \times \ldots \times V_{p}$, so

$$
\left|C_{\alpha}\right|\left\|B_{\alpha}\right\|_{V} \leq C^{\prime \prime} \prod_{j=1}^{p}\left(\mu_{\alpha_{j}}^{j}\right)^{\varepsilon-\varepsilon^{2}-\varepsilon \theta_{j}+\theta_{j}-\theta_{j}^{\prime}}
$$

Since $\theta_{j}^{\prime}>\theta_{j}$, for $\varepsilon$ small enough we have $\varepsilon-\varepsilon^{2}-\varepsilon \theta_{j}+\theta_{j}-\theta_{j}^{\prime}=:-\delta_{j}<0$, for all $j$. Hence $\sum_{\alpha}\left|C_{\alpha}\right|\left\|B_{\alpha}\right\|_{V}$ is majorized by the series

$$
\sum_{\alpha} C^{\prime \prime}\left(\mu_{\alpha_{1}}^{1}\right)^{-\delta_{1}} \ldots\left(\mu_{\alpha_{p}}^{p}\right)^{-\delta_{p}}=C^{\prime \prime} \prod_{j=1}^{p} \sum_{k=0}^{\infty}\left(\mu_{k}^{j}\right)^{-\delta_{j}}<\infty
$$

(here we use Proposition 3(i)).

3.4. Put $g(x)=\sum_{\alpha} C_{\alpha} B_{\alpha}(x), x \in \widehat{\Gamma}^{\prime}$. The real analyticity of $g$ in $\widehat{\Gamma}^{\prime}$ can be proved easily by using the local uniform convergence and Proposition 1; we also have the $\left(Q_{1}, \ldots, Q_{p}\right)$-analyticity of $g$ in $\widehat{\Gamma}^{\prime}$, i.e. for all $x \in \widehat{\Gamma}^{\prime}$ and 
$j=1, \ldots, p, g\left(\breve{x}_{j}, \cdot\right)$ satisfies

$$
Q_{j}\left(\frac{\partial}{\partial u_{1}}, \ldots, \frac{\partial}{\partial u_{N_{j}}}\right) g\left(\breve{x}_{j}, \cdot\right)=0 \quad \text { in }\left\{t \in \mathbb{C}:\left(\breve{x}_{j}, t\right) \in \widehat{\Gamma}^{\prime}\right\} .
$$

We now prove that $g=f$ on $\breve{E}_{j} \times D_{j}$ for all $j=1, \ldots, p$. We can suppose $j=p$ and that the property to be proved true for $p-1$ (induction hypothesis). For $x^{\prime}=\left(x_{1}, \ldots, x_{p-1}\right) \in E_{1} \times \ldots \times E_{p-1}$, the function $t \mapsto f\left(x^{\prime}, t\right)$ is $Q_{p}$-analytic in $D_{p} \ni D_{p}^{\prime}$, so it belongs to $L_{Q_{p}}^{2}\left(D_{j}^{\prime}\right)$ and we can write

$$
f\left(x^{\prime}, t\right)=\sum_{k} A_{k}\left(x^{\prime}\right) B_{k}^{p}(t)
$$

where the series converges uniformly on any compact set in $D_{j}^{\prime}$ and

$$
A_{k}\left(x^{\prime}\right)=\int_{E_{p}} f\left(x^{\prime}, v\right) \overline{B_{k}^{p}}(v) d \mu^{p}(v) .
$$

Put $x=\left(x^{\prime}, t\right)$ with $x^{\prime}=\left(x_{1}, \ldots, x_{p-1}\right)$ and $\alpha=\left(\alpha^{\prime}, k\right), \alpha^{\prime}=\left(\alpha_{1}, \ldots, \alpha_{p-1}\right)$. By the absolute convergence of $\sum_{\alpha} C_{\alpha} B_{\alpha}$ on $E_{1} \times \ldots \times E_{p}$, we can write

$$
g\left(x^{\prime}, t\right)=\sum_{k=0}^{\infty}\left(\sum C_{\alpha^{\prime}, k} B_{\alpha_{1}}^{1}\left(x_{1}\right) \ldots B_{\alpha_{p-1}}^{p-1}\left(x_{p-1}\right)\right) B_{k}^{p}(t)
$$

for $\left(x^{\prime}, t\right) \in E_{1} \times \ldots \times E_{p}$. We claim that

$$
\sum_{\alpha^{\prime}} C_{\alpha^{\prime}, k} B_{\alpha_{1}}^{1}\left(x_{1}\right) \ldots B_{\alpha_{p-1}}^{p-1}\left(x_{p-1}\right)=\int_{E_{p}} f\left(x^{\prime}, v\right) \overline{B_{k}^{p}}(v) d \mu^{p}(v) .
$$

In fact with the notation $B_{\alpha^{\prime}}(u)=B_{\alpha_{1}}^{1}\left(u_{1}\right) \ldots B_{\alpha_{p-1}}^{p-1}\left(u_{p-1}\right)$, we see that the left hand side is equal to

$$
\int_{E_{p}}\left(\sum_{\alpha^{\prime}}\left(\int_{\breve{E}_{p}} f(\cdot, v) \bar{B}_{\alpha^{\prime}} d\left(\mu^{1} \otimes \ldots \otimes \mu^{p-1}\right)\right) B_{\alpha^{\prime}}\left(x^{\prime}\right)\right) \overline{B_{k}^{p}}(v) d \mu^{p}(v) .
$$

By the induction hypothesis and paragraph 3.3, we have

$$
f(\cdot, v)=\sum_{\alpha^{\prime}}\left(\int_{\breve{E}_{p}} f(\cdot, v) \bar{B}_{\alpha^{\prime}} d\left(\mu^{1} \otimes \ldots \otimes \mu^{p-1}\right)\right) B_{\alpha^{\prime}} .
$$

with absolute uniform convergence on $\breve{E}_{p}$.

Now (5) and (4) give (3).

Finally (3), (2) and (1) give

$$
f\left(x^{\prime}, t\right)=g\left(x^{\prime}, t\right), \quad \forall t \in E_{p}, \forall x^{\prime} \in \breve{E}_{p} .
$$

Because $f\left(x^{\prime}, t\right)$ and $g\left(x^{\prime}, t\right)$ are $Q_{p}$-analytic functions of $t$ in the domain $D_{1}^{\prime}$, and $E_{p}$ is $L$-regular in $\mathbb{C}^{N_{p}}$, we conclude that $f\left(x^{\prime}, t\right)=g\left(x^{\prime}, t\right)$ on $\breve{E}_{p} \times D_{p}^{\prime}$.

Because of the arbitrariness of $D_{1}^{\prime}, \ldots, D_{p}^{\prime}$, the theorem is true. 
REmarks. We conjecture that the result is true if instead of the $L$ regularity of $E_{j}$, we suppose that $E_{j}$ satisfies the Leja condition for $Q_{j^{-}}$ analytic polynomials. This is proved in the following cases:

- $N_{j}=2, Q_{j}(D)=$ Laplacian (Zeriahi [12]).

- $p=2$ (Hécart [3], [4]).

\section{References}

[1] D. H. Armitage, T. Bagby and P. M. Gauthier, Note on the decay of elliptic functions, Bull. London Math. Soc. 17 (1985), 554-556.

[2] J. M. Hécart, Extremal functions for harmonic functions, Vietnam J. Math. 27 (1999), 53-59.

[3] - Fonctions séparément solutions d'une équation aux dérivées partielles, thèse de doctorat de l'Univ. Paul Sabatier, Toulouse, 1998.

[4] - Ouverts d'harmonicité pour les fonctions séparément analytiques, Potential Anal. 13 (2000), 115-126.

[5] M. Klimek, Pluripotential Theory, London Math. Soc. Monographs 6, Clarendon Press, 1991.

[6] N. Levenberg, Monge-Ampère measures associated to extremal plurisubharmonic functions in $\mathbb{C}^{n}$, Trans. Amer. Math. Soc. 289 (1985), 333-343.

[7] B. Mityagin, Approximative dimension and bases in nuclear spaces, Russian Math. Surveys 16 (1961), 59-127.

[8] Nguyen Thanh Van, Condition polynômiale de Leja et L-régularité dans $\mathbb{C}^{n}$, Ann. Polon. Math. 46 (1985), 237-241.

[9] Nguyen Thanh Van et J. Siciak, Fonctions plurisousharmoniques extrémales et systèmes doublement orthogonaux de fonctions analytiques, Bull. Sci. Math. 115 (1991), 235-244.

[10] Nguyen Thanh Van et A. Zeriahi, Systèmes doublement orthogonaux de fonctions holomorphes et applications, in: Topics in Complex Analysis, Banach Center Publ. 31, Inst. Math., Polish Acad. Sci., 1995, 281-297.

[11] V. P. Zahariuta, Spaces of harmonic functions, in: Functional Analysis, Transl. Math. Monographs 134, Amer. Math. Soc. 1994.

[12] A. Zeriahi, Bases communes dans certains espaces de fonctions harmoniques et fonctions séparément harmoniques sur certains ouverts de $\mathbb{C}^{n}$, Ann. Fac. Sci. Toulouse 4 (1982), 75-102.

Laboratoire de Mathématiques E. Picard

UMR CNRS 5580

Université Paul Sabatier

118 route de Narbonne

31062 Toulouse, France

E-mail: nguyen@picard.ups-tlse.fr 